\title{
ARHGAP29 expression may be a novel prognostic factor of cell proliferation and invasion in prostate cancer
}

\author{
KOSUKE SHIMIZU $^{1}$, HIROAKI MATSUMOTO ${ }^{1}$, HIROSHI HIRATA ${ }^{1,2}$, KOJI UENO $^{3}$, MASAHIRO SAMOTO ${ }^{1}$, \\ JUNICHI MORI $^{1}$, NAKANORI FUJII ${ }^{4}$, YOSHIHISA KAWAI ${ }^{5}$, RYO INOUE ${ }^{1}$, YOSHIAKI YAMAMOTO ${ }^{1}$, \\ SEIJI YANO $^{1}$, TOMOYUKI SHIMABUKURO ${ }^{1,6}$, MAKOTO FURUTANI-SEIKI ${ }^{7}$ and HIDEYASU MATSUYAMA ${ }^{1}$ \\ ${ }^{1}$ Department of Urology, Graduate School of Medicine, Yamaguchi University, Ube, Yamaguchi 755-8505; \\ ${ }^{2}$ Department of Urology, Ube Memorial Hospital, Ube, Yamaguchi $755-0051$; ${ }^{3}$ Center for Regenerative Medicine, \\ Graduate School of Medicine, Yamaguchi University, Ube, Yamaguchi 755-8505; ${ }^{4}$ Department of Urology, \\ Japan Community Health Care Organization Tokuyama Central Hospital, Shunan, Yamaguchi 745-8522; \\ ${ }^{5}$ Kawai Urological Clinic, Hofu, Yamaguchi 747-0836; ${ }^{6}$ Department of Urology, Ube Industries Central Hospital, \\ Ube, Yamaguchi 755-0151; ${ }^{7}$ Department of Systems Biochemistry in Pathology and Regeneration, \\ Graduate School of Medicine, Yamaguchi University, Ube, Yamaguchi 755-8505, Japan
}

Received July 15, 2019; Accepted June 23, 2020

DOI: $10.3892 /$ or.2020.7811

\begin{abstract}
Yes-associated protein (YAP) is a transcription-coupling factor that plays a central role in the Hippo pathway, and its activation regulates cell proliferation and carcinogenesis. YAP activation has been reported in various malignancies, conferring tumors with migratory and invasive abilities. Several studies have suggested that YAP expression is closely associated with prostate cancer. Furthermore, YAP has been revealed to regulate destabilization of F-actin associated with the cytoskeleton via Rho GTPase-activating protein 29 (ARHGAP29), suggesting that ARHGAP29 is associated with cancer metastasis. In the present study, the functions of ARHGAP29 were examined in four prostate cancer cell lines (22Rv1, LNCaP, DU145 and PC-3) and it was revealed that upregulation of ARHGAP29 in LNCaP and DU145 cells with the lowest expression of ARHGAP29 promoted cell proliferation and invasion. Conversely, ARHGAP29 knockdown in PC-3 cells with its highest expression level significantly reduced cell proliferation and invasion. In addition, immunohistochemistry of specimens from 133 patients who underwent radical prostatectomy was performed to investigate the clinical association between ARHGAP29 expression and prognosis in prostate cancer patients. Multivariate analysis demonstrated that ARHGAP29 was an independent prognostic factor for biochemical progression-free survival $(\mathrm{P}=0.0123)$. These
\end{abstract}

Correspondence to: Professor Hideyasu Matsuyama, Department of Urology, Graduate School of Medicine, Yamaguchi University, 1-1-1 Minami-kogushi, Ube, Yamaguchi 755-8505, Japan

E-mail: hidde@yamaguchi-u.ac.jp

Key words: Yes-associated protein, ARHGAP29, prostate cancer, LNCaP, PC-3 findings indicated that ARHGAP29 in prostate cancer may be a potential prognostic biomarker and therapeutic target.

\section{Introduction}

Prostate cancer was the second leading cause of cancer incidence (13.5\% of approximately 9.5 million new cases) and the fifth leading cause of mortality (6.7\% of approximately 5.4 million deaths) globally in 2018 for males according to Globocan (1). Advanced or metastatic prostate cancer patients usually respond well to initial androgen deprivation therapy (ADT). However, ADT does not prevent progression of prostate cancer despite the maintenance of low levels of testosterone over an extended period of time. Disease at this stage is termed castration-resistant prostate cancer (CRPC). Several systemic agents have been approved for the treatment of CRPC. However, despite the significant development of treatment options, CRPC remains as a lethal disease (2).

Genomic aberrations are common in prostate cancer cells. Various oncogenes and tumor suppressor genes are related to prostate cancer (3-8). PTEN, a tumor suppressor gene $(9,10)$, regulates androgen receptor (AR) signaling (11) in prostate cancer. A change in AR signaling is associated with the acquisition of castration resistance in prostate cancer (12). However, the mechanism of prostate cancer progression is still not completely understood.

Since the 1990s, the Hippo signaling pathway has been revealed as a tumor suppressor signaling pathway. Yes-associated protein (YAP) plays a central role in the Hippo pathway and has been revealed to regulate cell proliferation, migration, and invasion in various cancers including prostate cancer (13). High expression of YAP has been revealed to be associated with the differentiation and extra-prostatic extension of prostate cancer $(14,15)$. YAP expression has also been revealed to be associated with castration-resistant growth of prostate cancer cells as well as proliferation of 
androgen-independent human prostate cancer cells $(13,16)$. It is also known that YAP is activated by mechano-transduction via the hardness of the ECM (17).

YAP was revealed to bind to certain Rho GTPase-activating proteins (ARHGAPs), resulting in cytoskeletal rearrangement and the promotion of cell migration by altering the dynamics of F-actin/G-actin turnover in gastric cancer $(18,19)$. Furthermore, some ARHGAPs are regarded as effectors of YAP $(17,18)$. Thus far, the function of Rho GTPase-activating protein 29 (ARHGAP29) has been unclear in prostate cancer. Therefore, YAP and ARHGAP29 were examined, to investigate the role of ARHGAP29 in prostate cancer.

The aim of this study was to elucidate the role of ARHGAP29 by in vitro analysis and determine whether its protein expression is associated with prostate cancer prognosis.

\section{Materials and methods}

Patients. In total, 133 patients who underwent radical prostatectomy at Yamaguchi University Hospital from November 2000 to September 2016 were enrolled in the present study. All patients were diagnosed pathologically with prostate cancer. Detailed patient characteristics are presented in Table I. The present study was approved by the Institutional Ethics Committee of the Graduate School of Medicine of Yamaguchi University and written informed consent was obtained from all individuals enrolled in the study.

Immunohistochemistry.Formalin-fixedandparaffin-embedded tissue specimens were subjected to H\&E staining and immunohistochemical (IHC) staining. For each sample, $3-\mu \mathrm{m}$-thick sections were deparaffinized in xylene, dehydrated in ethanol, and incubated in a $0.3 \%$ hydrogen peroxide solution in methanol for $10 \mathrm{~min}$ at room temperature. The sections were then microwaved in a $0.01 \mathrm{M}$ citrate-buffered solution ( $\mathrm{pH}$ 6.0) for $15 \mathrm{~min}$ and covered in blocking solution (IMMUNO SHOT; Cosmo Bio Co., Ltd.) for $30 \mathrm{~min}$ at room temperature. Then, a primary antibody [anti-ARHGAP29 (1:200 dilution; cat. no. HPA026534; Atlas Antibodies) or anti-YAP (1:200 dilution; product no. 14074; Cell Signaling Technology, Inc.)] was incubated according to the manufacturers' instructions overnight at $4^{\circ} \mathrm{C}$, followed by incubation with the respective secondary antibody (N-Histofine Simple Stain MAX PO MULTI; cat. no. 414152F; Nichirei Biosciences, Inc.) for $30 \mathrm{~min}$ at room temperature. To evaluate IHC staining, the $\mathrm{H}$-score was used in the present study. Briefly, $>500$ tumor cells were counted in five different fields of vision in each section (x100, magnification), and the $\mathrm{H}$-score was calculated by multiplying the percentage of positive cells by the intensity (strongly stained, 3x; moderately stained, $2 \mathrm{x}$; weakly stained, $1 \mathrm{x}$ ), yielding a possible range of 0-300 (20-22). Two independent examiners (KS and HM) judged the scores and the mean score was set to the representative score. Cut-off of the H-score was determined by receiver operating characteristic (ROC) curve.

Cell lines. Four primary prostate cancer cell lines (22Rv1, ATCC no. CRL-2505; LNCaP, ATCC no. CRL-1740; DU145, ATCC no. HTB-81; PC-3, ATCC no. CRL-1435) were purchased from the American Type Culture Collection. Cells were cultured in RPMI-1640 and DMEM (Life Technologies;
Table I. Characteristics of 133 patients who underwent radical prostatectomy.

\begin{tabular}{lc}
\hline Characteristics & $\mathrm{n}(\%)$ \\
\hline Age in years, median (range) & $67(54-76)$ \\
Initial PSA, median (range) & $8.69 \mathrm{ng} / \mathrm{ml}$ \\
& $(3.53-354 \mathrm{ng} / \mathrm{ml})$ \\
Clinical T category & \\
$\leq \mathrm{T} 1 \mathrm{c}$ & $30(23)$ \\
T2a & $21(16)$ \\
T2b & $60(45)$ \\
T2c & $17(13)$ \\
$\geq \mathrm{T} 3$ & $5(4)$ \\
D'Amico risk classification & \\
Low & $15(11)$ \\
Intermediate & $55(41)$ \\
High & $63(47)$ \\
Gleason score & \\
$\leq 6$ & $33(25)$ \\
7 & $65(49)$ \\
$\geq 8$ & $35(26)$ \\
Preoperative ADT & \\
Yes & $35(25)$ \\
No & $98(75)$ \\
\hline
\end{tabular}

PSA, prostate specific antigen; ADT, androgen deprivation therapy.

Thermo Fisher Scientific, Inc.) supplemented with $10 \%$ fetal bovine serum (Biological Industries) and maintained in humidified incubators with $5 \% \mathrm{CO}_{2}$ at $37^{\circ} \mathrm{C}$.

siRNA knockdown of ARHGAP29. si-ARHGAP29 and control siRNAs were obtained from Life Technologies; Thermo Fisher Scientific, Inc. siRNA sequences were as follows: ARHGAP29-\#1 siRNA sense, 5'-GCAUAGGUGUUGUUG AUCAtt-3' and antisense, 5'-UGAUCAACAACACCUAUG Cta-3'; ARHGAP29-\#2 siRNA sense, 5'-GACCAAGGCUAA AACGAAUtt-3' and antisense, 5'-AUUCGUUUUAGCCUU GGUCtc-3'. The PC-3 cell line was transiently transfected with siRNA using Lipofectamine RNAi MAX (Thermo Fisher Scientific, Inc.), according to the manufacturer's instructions. After transfection, cells were incubated at $37^{\circ} \mathrm{C}$ in a $\mathrm{CO}_{2}$ incubator for $48 \mathrm{~h}$. Quantitative evaluations of mRNA and protein expression were performed by western blotting and RT-qPCR, respectively.

Plasmid construction and transfection. A mammalian expression of HA tagged ARHGAP29 (\#104154) was purchased from Addgene, Inc. Cells were seeded on culture dishes at density of $1 \times 10^{5} /$ well in a 6 -well plate, and the pcDNA3.1 empty vector plasmid (mock) (Thermo Fisher Scientific, Inc.) or $2 \mu \mathrm{g}$ of ARHGAP29 expressing plasmid were transfected using X-tremeGENE HP DNA transfection Reagent (Sigma-Aldrich; Merck KGaA) for $48 \mathrm{~h}$, according to the manufacturer's instructions. 
Regarding the DU145 cell line, plasmid transfection was performed via electroporesis system using an Amaxa cell line Nucleofector Kit L (cat. no. VACA-1005; Lonza Group, Ltd.) according to the manufacturer's instructions. Prior to electroporation, $1 \times 10^{6}$ DU145 cells were centrifuged at $90 \times \mathrm{g}$ for $5 \mathrm{~min}$, resuspended in $100 \mu \mathrm{l}$ of Nucleofector solution and mixed with $2 \mu \mathrm{g}$ of pmaxGFP or $2 \mu \mathrm{g}$ of ARHGAP29 plasmid. The aforementioned cells were transferred to cuvettes and immediately electroporated based on the DU145 program (Nucleofector Program A-023) using Nucleofector 2b Device. After electroporation, cells were incubated in the cuvette at room temperature for $10 \mathrm{~min}$ and then $500 \mu \mathrm{l}$ of pre-warmed RPMI-1640 medium supplemented with $10 \%$ FBS were added to the cuvette. Cells were transferred to a 6 -well plate and incubated at $37^{\circ} \mathrm{C} 5 \% \mathrm{CO}_{2}$ overnight. The day after electroporation, cells were centrifuged and the medium was replaced by RPMI-1640 supplemented with $10 \%$ FBS and incubated for $48 \mathrm{~h}$ and then performed subsequent experiments were performed.

Reverse transcription quantitative PCR (RT-qPCR). We created cDNA by reverse transcription of mRNAs extracted from each prostate cancer cell line (22Rv1, LNCaP, DU145 and PC-3), using iScript Advanced cDNA Synthesis Kit for RT-qPCR (cat. no. 1725037; Bio-Rad Laboratories, Inc.). Quantitative real-time RT-PCR was performed in triplicate with an Applied Biosystems StepOnePlus using TaqMan universal PCR master mix (Applied Biosystems; Thermo Fisher Scientific, Inc.), according to the manufacturer's protocols. The TaqMan probes and primers were purchased from Applied Biosystems. Human GAPDH (assay ID: 02786624) was used as an endogenous control. Levels of ARHGAP29 (assay ID: 00191351) and MMP-2 (assay ID: 01548727) RNA expression were determined using StepOnePlus software (version 2.2.2; Applied Biosystems; Thermo Fisher Scientific, Inc.). The miRNA expression levels were determined using the $2^{-\Delta \Delta \mathrm{Cq}}$ method (23). The cycling conditions consisted of an initial denaturation at $95^{\circ} \mathrm{C}$ for $30 \mathrm{sec}$ and PCR at 40 cycles at $95^{\circ} \mathrm{C}$ for $5 \mathrm{sec}$ and $60^{\circ} \mathrm{C}$ for $30 \mathrm{sec}$.

Gene expression analysis by $q P C R$. Total RNA was isolated from cells (PC-3-si-NC and si-ARHGAP29), and an RT ${ }^{2}$ Profiler PCR Array (Qiagen RT ${ }^{2}$ Profiler PCR Array Human Cell Motility; cat. no. PAHS-128Z, product no. 330231) was used to examine the expression patterns of genes involved in human cell motility, according to the manufacturer's instructions. We analyzed the gene expression levels and produced a heatmap using the web-based software 'RT2 Profiler PCR Array' Data Analysis version 3.5 (Qiagen, Inc.).

Western blotting. Cells samples were lysed in RIPA buffer (cat. no. 89900; Thermo Fisher Scientific, Inc.) supplemented with $1 \%$ protease inhibitors (cOmplete ${ }^{\mathrm{TM}}$, Mini,cat.no.04693124001; Sigma-Aldrich) for total protein extraction. We quantified the concentration of total proteins using BCA. Each lysate sample (30 $\mu \mathrm{g}$ protein) was separated by $4-20 \%$ SDS-PAGE (Mini-PROTEAN TGX Stain-Free Gels, cat. no. 4568095; Bio-Rad Laboratories), and then electro-transferred to a PVDF membrane. After blocking in 5\% dry non-fat milk or 5\% BSA for $1 \mathrm{~h}$ at room temperature, the membranes were incubated with a primary antibody overnight at $4^{\circ} \mathrm{C}$. After washing in
TBS with $0.05 \%$ Tween-20 (TBST), the membranes were incubated with an HRP-conjugated secondary antibody for $1 \mathrm{~h}$ at room temperature. After washing with TBST, signals were detected using an ECL detection system (ChemiDoc ${ }^{\text {TM }}$ XRS+; Bio-Rad Laboratories, Inc). Primary antibodies were as follows: anti-ARHGAP29 (product code ab85853, 1:2,000 dilution), anti-AR (product code ab133273, 1:1,000 dilution), anti-F-actin (product code ab205, 1:500 dilution) and anti-MMP-2 (product code ab97779, 1:1,000 dilution) from Abcam and anti-YAP (cat. no. 14074S; 1:1,000 dilution), anti-phosphorylated YAP (cat. no. $13008 \mathrm{~S} ; 1: 1,000$ dilution), anti-GAPDH (cat. no. 5174S; 1:1,000 dilution), anti-Cofilin (cat. no. 5175T; 1:1,000 dilution) and anti-phospho-Cofilin (cat. no. 3313T; 1:1,000 dilution) were obtained from Cell Signaling Technology Inc. Secondary antibodies were as follows: goat anti-rabbit IgG H\&L (HRP) (product code ab6721; 1:10,000 dilution) and goat anti-mouse IgG H\&L (HRP) (product code ab6789; 1:10,000 dilution) from Abcam. GAPDH was used for protein normalization. We performed densitometry using the public domain free software ImageJ (version 1.51; National Institutes of Health).

Cell viability and invasion assays. Cell viability was assessed using an MTS assay (CellTiter $96 \mathrm{AQ}_{\text {ueous }}$ One Solution Cell Proliferation Assay; Promega Corporation). After the cells were seeded at density of $5 \times 10^{3} /$ well in a 96 -well plate, cell viability was measured at 24,48, and $72 \mathrm{~h}$ at an OD of $490 \mathrm{~nm}$. Data are expressed as the mean \pm SD of three independent experiments. Cell invasion assays were performed using a CytoSelect 24-well cell invasion assay kit (Cell BioLabs, Inc.). The CytoSelect ${ }^{\mathrm{TM}}$ Cell Invasion Assay Kit contains polycarbonate membrane inserts $(8-\mu \mathrm{m}$ pore size) in a 24 -well plate. The upper surface of the insert membrane is coated with a uniform layer of dried basement membrane matrix solution. This basement membrane layer serves as a barrier to discriminate invasive cells from non-invasive cells. A cell suspension containing $0.5-1.0 \times 10^{6}$ cells $/ \mathrm{ml}$ was placed in upper chamber in serum-free media. A total of $500 \mu \mathrm{l}$ of media containing $10 \%$ fetal bovine serum was added to the lower well of the invasion plate. After $48 \mathrm{~h}$ of incubation at $37^{\circ} \mathrm{C}$ with $5 \% \mathrm{CO}_{2}$, cells invaded through the basement membrane layer and clung to the bottom of the insert membrane. Non-invasive cells remained in the upper chamber. After removal of non-invasive cells, invasive cells were stained for $10 \mathrm{~min}$ at room temperature using Cell Stain Solution (Part no. 11002; CytoSelect 24-well cell invasion assay kit) and then quantified. Each insert was transferred to an empty well, $200 \mu \mathrm{l}$ of Extraction Solution (Part no. 11003; CytoSelect 24-well cell invasion assay kit) was added per well and then incubation followed for $10 \mathrm{~min}$ on an orbital shaker. Subsequently $100 \mu \mathrm{l}$ from each sample was transferred to a 96-well microtiter plate and the OD 560 $\mathrm{nm}$ of each sample was measured on a plate reader, according to the manufacturer's instructions.

Database. The Cancer Genome Atlas (TCGA) accessed from the data portal of the National Cancer Institute Home Page (http://cancergenome.nih.gov/) was used for comparison with our data.

Statistical analysis. Categorical variables were compared by the Chi-squared test. Continuous variables were analyzed 
A

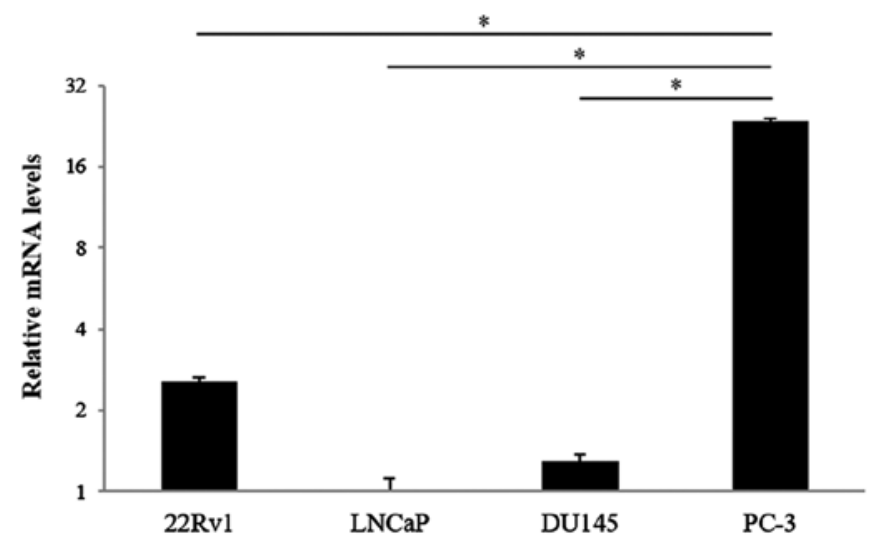

B

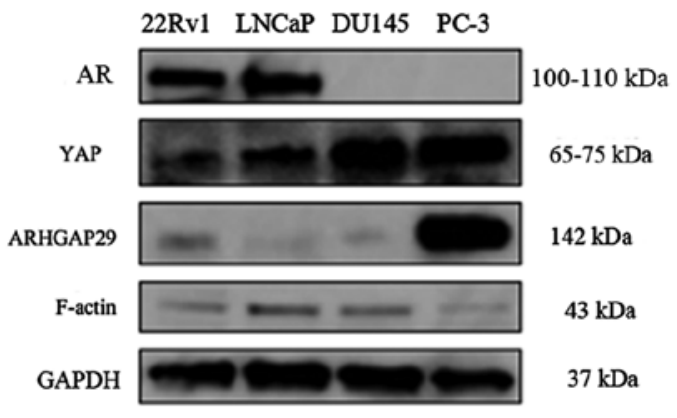

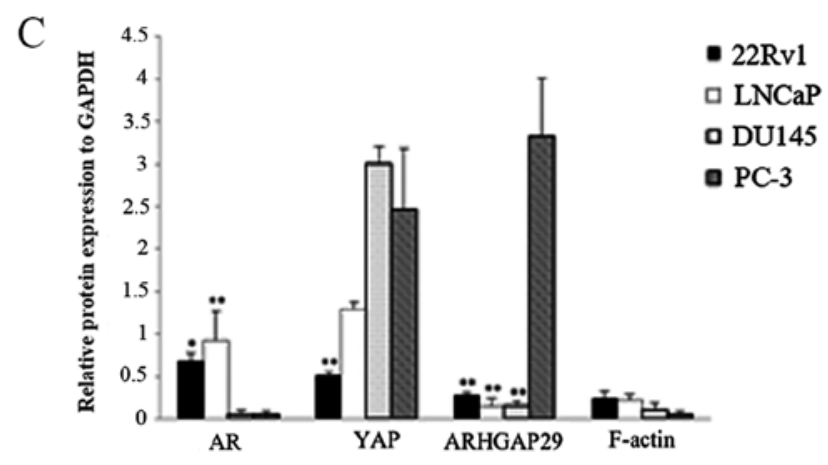

Figure 1. AR, YAP, ARHGAP29, and F-actin expression in prostate cancer cell lines. (A) mRNA levels of ARHGAP29 in prostate cancer cell lines (22Rv1, LNCaP, DU145 and PC-3). Experiments were performed in triplicate. The vertical axis of the graph is presented on a logarithmic scale. The results are expressed as the mean \pm SD. " $\mathrm{P}<0.01$ compared with PC-3 cells. (B) Western blotting of the expression of various proteins in prostate cancer cell lines. (C) Densitometric analysis of B (relative protein expression to GAPDH). There was an inverse association between ARHGAP29 and F-actin expression, but no clear association between the expression of ARHGAP29 and that of other proteins. The results are expressed as the mean $\pm \mathrm{SD}$ (at least three independent experiments). "P $<0.05$, ${ }^{* *} \mathrm{P}<0.01$ compared with PC-3 cells. AR, androgen receptor; YAP, yes-associated protein; ARHGAP29, Rho GTPase-activating protein 29.

using the unpaired Student's t-test when comparing two groups. One-way ANOVA followed by Tukey-Kramer test were used when comparing more than two groups. Survival analysis was estimated by the Kaplan-Meier method and compared by the log-rank test. A Cox proportional hazards regression model was used in the multivariable analysis to identify risk factors for disease progression. Statistical analysis was performed using JMP software (Pro.13; SAS Institute). P-values were two-sided, and statistical significance was defined as $\mathrm{P}<0.05$ in all tests. Regarding protein expression, bivariate analysis was performed and a ROC curve was constructed using JMP software to set the cutoff value and determine the high/low expression of proteins (24).

\section{Results}

AR, YAP, ARHGAP29, and F-actin expression in prostate cancer cell lines. RT-qPCR and western blotting were performed to clarify whether there was a difference in the expression of ARHGAP29 between prostate cancer cell lines depending on AR and YAP expression. AR was expressed in 22Rv1 and LNCaP cell lines. YAP was expressed in all four prostate cancer cell lines (Fig. 1A and B). The expression level of YAP was higher in DU-145 and PC-3 cells than in LNCaP and 22Rv1 cells. ARHGAP29 protein expression was higher in PC-3 cells than in the other cell lines. F-actin was the most weakly expressed in PC-3 cells compared with the other cell lines (Fig. 1C).

Effect of downregulation or upregulation of ARHGAP29 in prostate cancer cell lines (PC-3, LNCaP and DU145). After downregulation (PC-3 cells) or upregulation (LNCaP and DU145 cells) of ARHGAP29 in prostate cancer cell lines (Fig. 2A and B), the expression of several proteins was examined. Based on a recent study (18), the RhoA-LIMK-cofilin signaling pathway has been revealed to be affected by ARHGAP29 in a gastric cancer cell line. Therefore, certain related genes (cofilin, p-cofilin and F-actin) were analyzed by western blotting (Fig. 2B).

After almost complete knockdown of ARHGAP in PC-3 cells, phosphorylated cofilin and F-actin were increased and cofilin expression was unchanged. In contrast, after overexpression of ARHGAP29 in DU145 cells, F-actin was slightly decreased but phosphorylated cofilin was not altered. YAP and phosphorylated YAP were slightly recovered without significant differences in si-ARHGAP29 PC-3 transfectants compared with the si-NC control. Conversely, YAP was decreased after overexpression of ARHGAP29 in DU145 cells. Expression of these proteins relative to that of the housekeeping gene GAPDH and the ratio of phosphorylated protein 
A

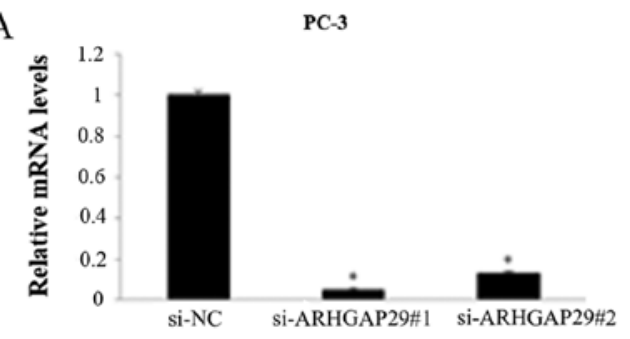

B $\quad$ PC-3
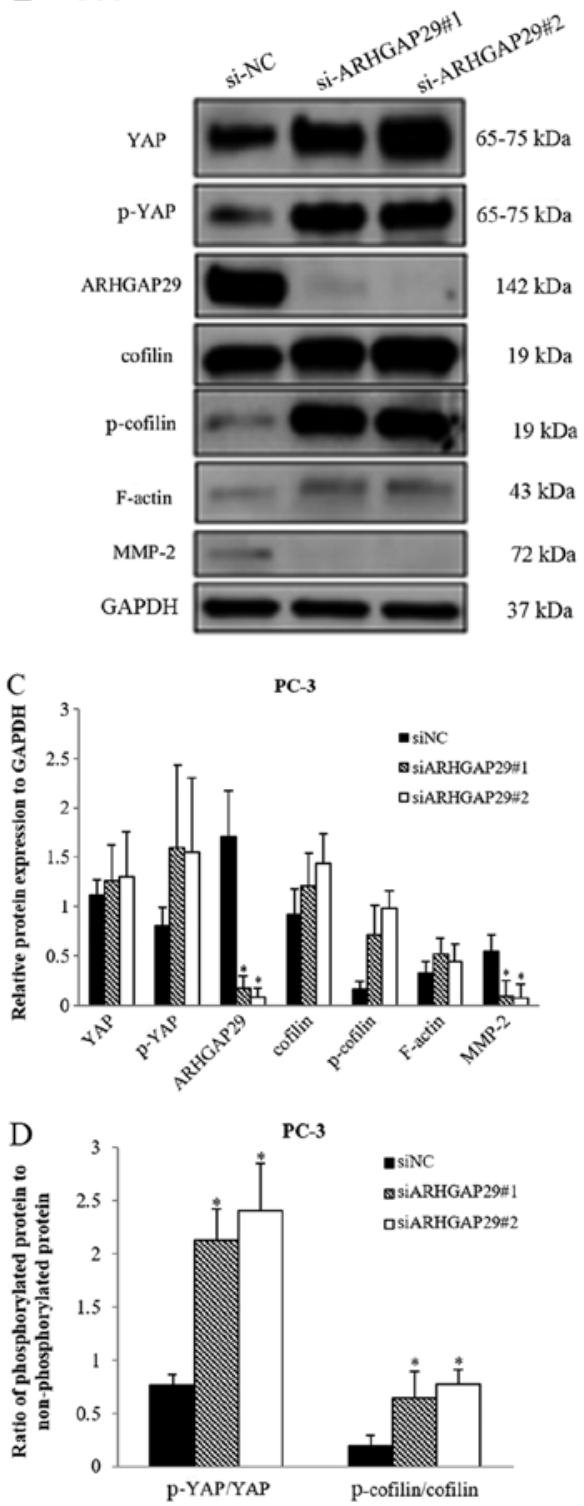

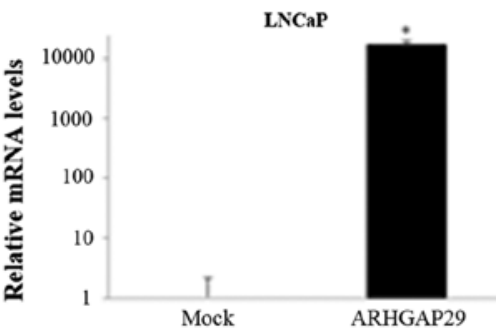

LNCaP
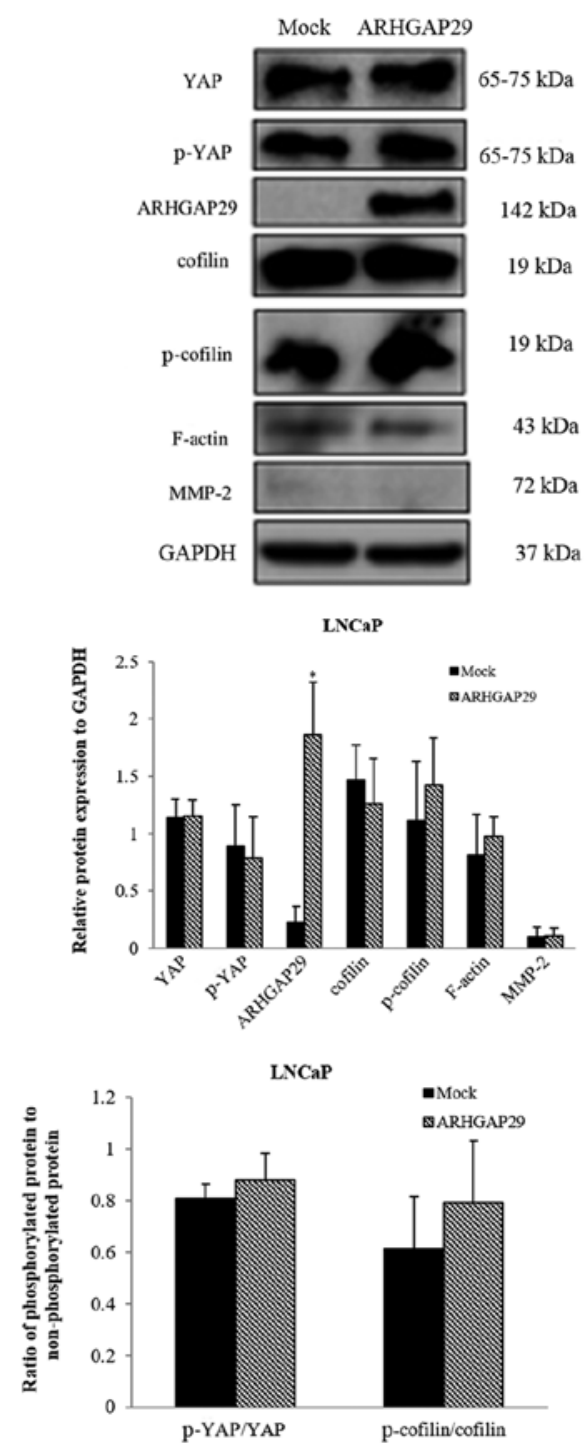

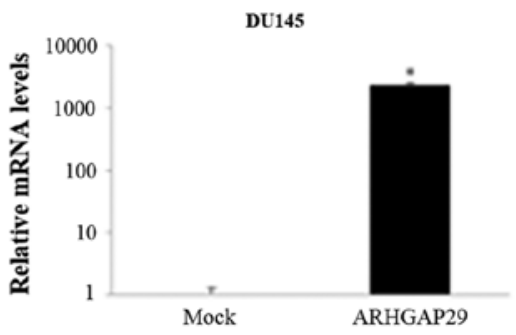

DU145

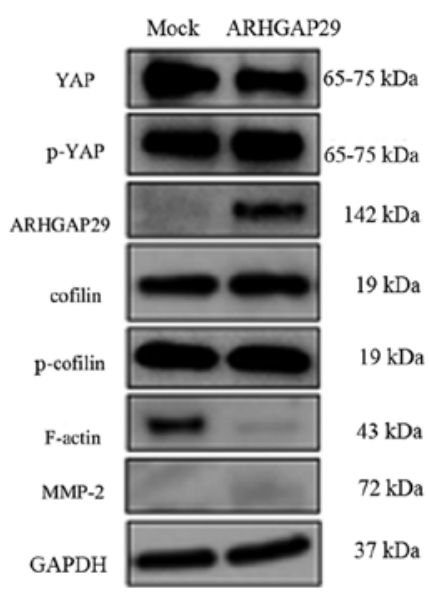

DU145

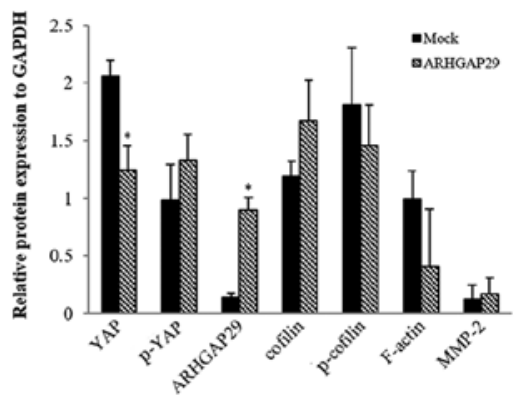

DU145

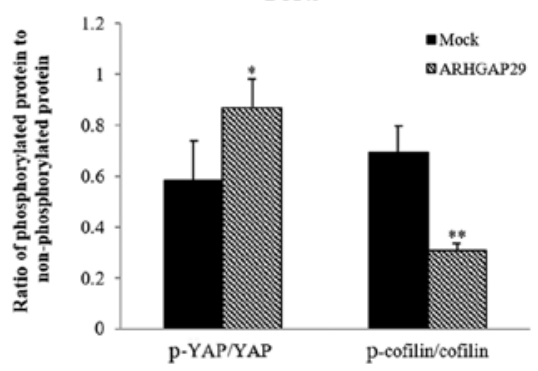

Figure 2. Effects of downregulation or upregulation of ARHGAP29 in prostate cancer cell lines (PC-3, LNCaP and DU145) on the mRNA and protein expression. (A) Quantitative comparison of ARHGAP29 expression by RT-qPCR in prostate cancer cell lines with downregulation or upregulation of ARHGAP29 compared with the control. Experiments were performed in triplicate. The results are expressed as the mean $\pm \mathrm{SD}$. " $\mathrm{P}<0.01$ compared with si-NC (PC-3 cells) or pcDNA empty vector (mock; LNCaP and DU145 cells). In LNCaP and DU145, the vertical axis of the graph is presented on a logarithmic scale. (B) Western blotting of the expression of various proteins in prostate cancer cell lines. (C) Densitometric analysis of B (relative protein expression to GAPDH). The protein expression was only slightly altered in ARHGAP29-upregulated LNCaP and DU145 cells compared with ARHGAP29-downregulated PC-3 cells. The results are expressed as the mean $\pm \mathrm{SD}$ (at least three independent experiments). ${ }^{*} \mathrm{P}<0.01$ compared with si-NC (PC-3 cells) or pcDNA empty vector (mock; LNCaP and DU145 cells). (D) The ratio of phosphorylated protein to non-phosphorylated protein (YAP and cofilin). The results are expressed as the mean \pm SD (at least three independent experiments). ${ }^{*} \mathrm{P}<0.05,{ }^{* *} \mathrm{P}<0.01$ compared with si-NC (PC-3 cells) or pcDNA empty vector (mock; LNCaP and DU145 cells). ARHGAP29, Rho GTPase-activating protein 29.

to non-phosphorylated protein (YAP and cofilin) are presented in Fig. 2C and D.

Functional analyses by MTS and cell invasion assays were also performed in these three cell lines (Fig. 3). Cell viability and invasion were significantly decreased after downregulation of ARHGAP29 in PC-3 cells (Fig. 3A and B). After upregulation of ARHGAP29 in LNCaP and DU145 cells, cell viability and invasion were significantly increased (Fig. 3A and B). 
A

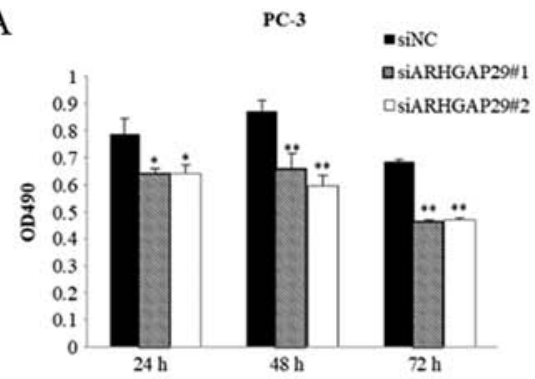

B

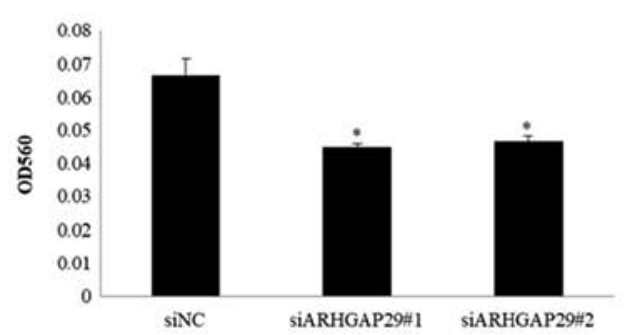

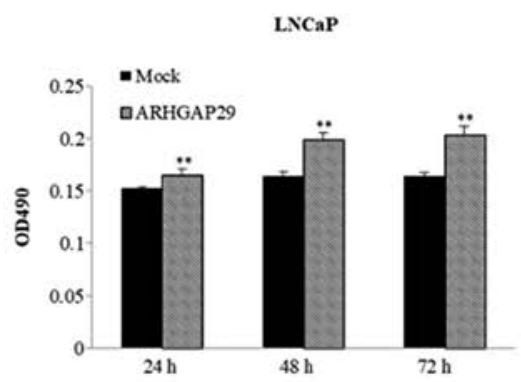

LNCaP

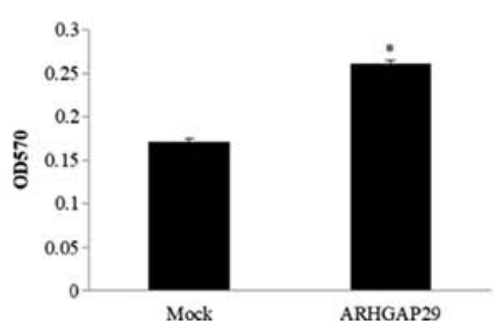

DU145

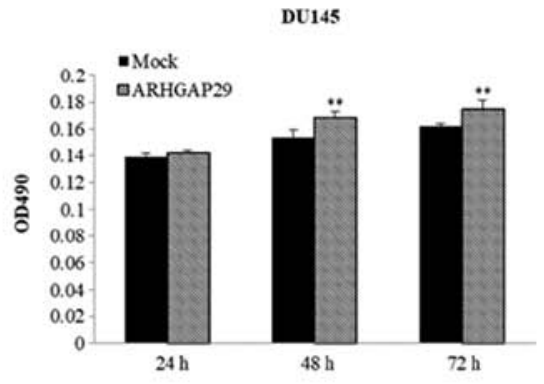

DU145

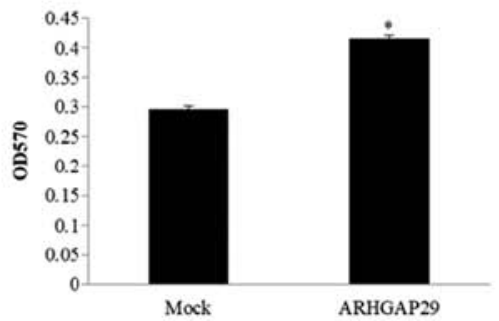

Figure 3. Effects of downregulation or upregulation of ARHGAP29 in prostate cancer cell lines (PC-3, LNCaP and DU145) on cell viability and invasion. (A) MTS assay results. Cell viability was significantly decreased in ARHGAP29-downregulated PC-3 cells compared with control cells (si-NC). In contrast, cell viability was significantly increased in ARHGAP29-upregulated LNCaP and DU145 cells compared with control cells (mock). Experiments were performed in triplicate. The results are expressed as the mean $\pm \mathrm{SD}$. ${ }^{*} \mathrm{P}<0.05,{ }^{* * *} \mathrm{P}<0.01$ compared with si-NC (PC-3 cells) or pcDNA empty vector (mock; LNCaP and DU145 cells). (B) Invasion assay results. The number of invasive PC-3 cells was significantly decreased after knocking down ARHGAP29. In contrast, the number of invasive LNCaP and DU145 cells was significantly increased after upregulation of ARHGAP29. Experiments were performed in triplicate. The results are expressed as the mean $\pm \mathrm{SD}$. ${ }^{*} \mathrm{P}<0.01$ compared with si-NC (PC-3 cells) or pcDNA empty vector (mock; LNCaP and DU145 cells). ARHGAP29, Rho GTPase-activating protein 29.

Identification of cell motility-related genes after knock down of ARHGAP29. Based on the functional analyses, ARHGAP29 may be involved in cell proliferation or invasion. To determine new therapeutic targets or genes related to ARHGAP29 in prostate cancer cells, Qiagen $\mathrm{RT}^{2}$ Profiler PCR Array Human Cell Motility was used.

The pre-designed array included 84 genes related to cell motility (Fig. 4A). Data analysis was performed using the web-based software 'RT2 Profiler PCR Array' Data Analysis version 3.5 as aforementioned. A heatmap is presented in Fig. 4B. When the boundary was set at 3, one gene (STAT3) was upregulated and numerous genes (including CSF1, ACTN3 and HGF) were downregulated after knocking down ARHGAP29 in PC3 cells (Fig. 4C). Regulation of some proteins, such as HGF, RHO, CAPN1, was validated by western blotting. However, there was no difference in the expression of these proteins between si-NC and si-ARHGAP29 cells (data not shown). Among the downregulated genes of the 84 genes in the array, active MMP2 expression was significantly decreased at mRNA and protein levels after knockdown of ARHGAP29 in PC3 cells (Figs. 2B and C and 4D).

Association between the expression level of ARHGAP29 and prognosis in prostate cancer patients. The expression level of ARHGAP29 was evaluated by IHC in 133 prostate cancer patients who had undergone radical prostatectomy. Representative images of YAP and ARHGAP29 staining in prostate cancer specimens (negative and positive) are presented Fig. 5A.
YAP expression was high in the nucleus of basal cells and the cytoplasm of luminal cells, but ARHGAP29 expression was high in the cytoplasm of both cells. Notably, YAP expression was unrelated to the Gleason score. The characteristics of the prostate cancer patients are presented in Table I. ARHGAP29 expression was significantly associated with the risk classification of prostate cancer (Fig. 5B). Both YAP and ARHGAP29 had low area under the curve (AUC) scores as prognostic markers, but there was a significant difference between the expression of these proteins and biochemical progression-free survival (b-PFS: $\mathrm{P}=0.0422$, and $\mathrm{P}=0.0123$, respectively) (Fig. 5C and D). In addition, high expression of both proteins was significantly associated with poor prognoses (Fig. 5D). In TCGA database, YAP did not exhibit a tendency for a poor prognosis in patients with high expression. In contrast, ARHGAP29 exhibited a tendency for a poor prognosis in patients with high expression in TCGA (Fig. S1A and B). Moreover, the prognostic significance of clinicopathological parameters, including prostate specific antigen (PSA), the D'Amico risk classification, Gleason score, and pathological $\mathrm{T}$ category, and the expression levels of YAP and ARHGAP29 were evaluated in prostate cancer patients (Table II). As a result, high ARHGAP29 expression was a significant independent risk factor related to b-PFS in multivariate analysis ( $\mathrm{HR}=2.27 ; \mathrm{P}<0.05$; data not shown).

\section{Discussion}

YAP has been revealed as an oncogenic protein in several cancers, such as gastric, breast, hepatocellular, pancreatic, and 
A

\begin{tabular}{|c|c|c|c|}
\hline & Negative & Si ARHGAP29 & \\
\hline & control Group-Ct & Test Group-Ct & gene information \\
\hline Al & 22.4033786 & 23.57656288 & A01 Hs.509765 NMI_001102 ACTN1 Actinin, alpha 1 \\
\hline $\mathrm{A}^{2}$ & 30.37800598 & 35.41236115 & A02 Hs.654432 NML 001104 ACTN3 Actinin, alpha 3 \\
\hline A3 & 24.39433861 & 25.62803459 & A03 Hs.270291 NMI 004924 ACTN4 Actinin, alpha 4 \\
\hline A4 & 23.25595474 & 25.58378601 & A04 Hs.728557 NML 005722 ACTR2 ARP2 actin-related protein 2 homolog (yeast) \\
\hline A5 & 21.28431892 & 23.55618477 & 405 Hs.433512 NM 005721 ACTR3 ARP3 actin-related protein 3 homolog (veast) \\
\hline A6 & 26.44383049 & 27.67854691 & $406 \mathrm{Hs} .525622 \mathrm{NM} 005163 \mathrm{AKT1}$ V-akt murine thymoma viral oncogene homolog 1 \\
\hline A7 7 & 23.9215641 & 25.69975908 & A07 Hs.525330 NM_ 001663 ARF 6 ADP-ribosylation factor 6 \\
\hline As & & & A08 Hs.159161 NM 004309 ARHGDIA Rho GDP dissociation inhibitor (GDD) alpha \\
\hline A 9 & 26.78385353 & & A09 Hs.508738 NM_ 003899 ARHGEF7 Rho guanine nucleotide exchange factor (GEF) 7 \\
\hline Al0 & 26.75171089 & & A10 Hs.128316 NM_006340 BAIAP2 BAIl-associated protein 2 \\
\hline All & 28.37572479 & 30.52178192 & All Hs.479747 NM 014567 BCARI Breast cancer anti-estrogen resistance 1 \\
\hline Al2 & 29.31593323 & 35.01529312 & A12 Hs.502842 NM_ 005186 CAPNI Calpain 1, (muI) large subunit \\
\hline Bl & 20.63972855 & 22.91611862 & B01 Hs.350599 NM 001748 CAPN2 Calpain 2, (m/I) large subunit \\
\hline B2 & 21.75957489 & 23.80058861 & B02 Hs.74034 NM 001753 CAV1 Caveolin 1, caveolae protein, 22kDa \\
\hline B3 & 22.65792656 & 24.68171501 & B03 Hs.690198 NM 001791 CDC42 Cell division cycle 42 (GTP binding protein, 25kDa) \\
\hline B4 & 20.99303627 & 22.61857224 & B04 Hs.170622 NMI 005507 CFL1 Cofilin 1 (non-muscle) \\
\hline B5 & 26.43111801 & 28.22467232 & B05 Hs.638121 NM 016823 CRK V-crk sarcoma virus CT10 oncogene homolog (avian) \\
\hline B6 & 31.2063961 & 35.56407166 & B06 Hs.591402 NM_ 000757 CSF1 Colony stimulating factor 1 (macrophage) \\
\hline B7 & 27.79785728 & 29.5569706 & B07 Hs.596164 NM 005231 CTIN Cortactin \\
\hline BS & 29.69989586 & 30.40603256 & B0S Hs.529451 NMI 005219 DIAPHI Diaphanous homolog 1 (Drosophila) \\
\hline B9 & 24.19006157 & 26.50961113 & B09 Hs.368912 NM_ 001935 DPP4 Dipeptidyl-peptidase 4 \\
\hline B10 & 23.56789017 & 27.92847252 & B10 Hs.419815 NMI 001963 EGF Epidermal growth factor \\
\hline B11 & 26.46381187 & 27.42577934 & B11 Hs.488293 NM 005228 EGFR Epidermal growth factor receptor \\
\hline B12 & 24.66299629 & 25.51127434 & B12 Hs.497893 NM_001008493 ENAH Enabled homolog (Drosophila) \\
\hline Cl & 25.76825714 & 26.54108047 & C01 Hs.487027 NM 003379 EZR Ezrin \\
\hline $\mathrm{C2}_{2}$ & 31.63132858 & 36.94598389 & C02 Hs.654370 NMI 004460 FAP Fibroblast activation protein, alpha \\
\hline C3 & 26.00258827 & 28.10666466 & C03 Hs.284244 NM_ 002006 FGF2 Fibroblast growth factor 2 (basic) \\
\hline C4 & 29.24377251 & 33.85067368 & C04 Hs.396530 NM_ 000601 HGF Hepatocyte growth factor (hepapoietin A; scatter factor) \\
\hline C5 & 30.44312477 & 34.8013649 & C05 Hs.160562 NM 000618 IGF1 Insulin-like growth factor 1 (somatomedin C) \\
\hline C6 & 30.43789482 & 31.03606033 & C06 Hs.643120 NM_ 000375 IGFIR Insulin-like growth factor 1 receptor \\
\hline $\mathrm{C7}$ & 26.16326904 & 27.19724846 & C07 Hs.5158 NM 004517 ILK Integrin-linked kinase \\
\hline C8 & 29.84774017 & 31.97459602 & C08 Hs.694732 NM 0008s5 ITGA4 Integrin, alpha 4 (antigen CD49D, alpha 4 subunit of VLA-4 receptor) \\
\hline C9 & 19.30079079 & 22.20329285 & C09 Hs.643813 NM_ 002211 ITGB1 Integrin, beta 1 (fibronectin receptor, beta polypeptide, antigen CD29 include MIDF2, MSK12) \\
\hline C10 & 30.20965195 & 33.98184204 & C10 Hs.375957 NM_ 000211 ITGB2 Integrin, beta 2 (complement component 3 receptor 3 and 4 subunit) \\
\hline C11 & 26.35469818 & 29.50634766 & C11 Hs.218040 NM_ 000212 ITGB3 Integrin, beta 3 (platelet glycoprotein IIIa, antigen CD61) \\
\hline C12 & 26.53396797 & 27.25556564 & C12 Hs.647035 NM_002314 LINKK1 LIMI domain kinase 1 \\
\hline Dl & 25.52104378 & 26.70186996 & D01 Hs.431850 NM 002745 MAPK1 Nitogen-activated protein kinase 1 \\
\hline D2 & 23.33166313 & 24.6860981 & D02 Hs.132966 NM 000245 NET Met proto-oncogene (hepatocyte growth factor receptor) \\
\hline D3 & 26.72789383 & 30.19018173 & D03 Hs.2399 NM 004995 MNIP14 Matrix metallopeptidase 14 (membrane-inserted) \\
\hline D4 & 30.91285943 & 35.19906235 & D04 Hs.513617 NM_ 004530 MMP2 Natrix metallopeptidase 2 (gelatinase A, 72kDa gelatinase, $72 \mathrm{kDa}$ type IV collagenase) \\
\hline D5 & 30.81827164 & 33.46358109 & D05 Hs.297413 NM 004994 MMP9 Matrix metallopeptidase 9 (gelatinase B, 92kDa gelatinase, 92kDa type IV collagenase) \\
\hline D6 & 23.89659309 & 25.46996689 & D06 Hs.87752 NM_ 002444 MSN MIoesin Position UniGene GenBank Symbol Description \\
\hline D7 & 28.58475876 & 29.5707283 & D07 Hs.16355 NM 005964 MYH10 Myosin, heavy chain 10, non-muscle \\
\hline Ds & 25.39417076 & 26.68235588 & D08 Hs.474751 NM 002473 MYH9 Myosin, heavy chain 9, non-muscle \\
\hline D9 & 22.90526797 & 27.61661148 & D09 Hs.504687 NM 006097 MYL9 My \\
\hline D10 & 27.46499634 & 29.14052391 & D10 Hs.477375 NM 053025 MYLK Mvos \\
\hline Dl1 & 25.69220543 & 28.26981163 & D11 Hs.435714 NM_ 002576 PAK1 P21 protein (Cdc42/Rac)-activated kinase I \\
\hline D12 & 27.82408142 & 29.27343178 & D12 Hs.20447 NM 005854 PAK4 P21 protein (Cdc42/Rac)-activated kinase 4 \\
\hline
\end{tabular}

\begin{tabular}{|c|c|c|c|}
\hline & Negative & Si ARHGAP29 & \\
\hline & control Group-Ct & Test Group-Ct & gene information \\
\hline E1 & 19.5062027 & 21.61876488 & E01 Hs.494691 NMI 005022 PFN1 Profilin 1 \\
\hline E2 & 23.45112038 & 26.43966293 & E02 Hs.553498 NM 006218 PIK3CA Phosphoinositide-3-kinase, catalytic, alpha polypeptide \\
\hline E3 & 24.82546043 & 26.42458725 & E03 Hs.466871 NM_ 002659 PLALR Plasminogen activator, urokinase receptor \\
\hline E4 & 27.84104919 & 29.60287476 & E04 Hs.268177 NM_002660 PLCG1 Phospholipase C, gamma I \\
\hline E5 & 26.54397392 & 29.31639481 & E05 Hs.382865 NM_ 002662 PLDI Phospholipase Dl, phosphatidylcholine-specific \\
\hline E6 & 25.01036835 & 26.59282875 & E06 Hs.531704 NM 002737 PRKCA Protein kinase C, alpha \\
\hline E7 & 32.08686829 & 36.71274567 & E07 Hs.500466 NM_ 000314 PTEN Phosphatase and tensin homolog \\
\hline ES & 24.70900726 & 26.6055069 & E0S Hs.395432 NM_ 005607 PTK2 PTK2 protein tyrosine kinase 2 \\
\hline E9 & 29.59366417 & 33.95464325 & E09 Hs.491322 NM_ 004103 PTK2B PTK2B protein tyrosine kinase 2 beta \\
\hline E10 & 29.94009399 & 30.88469505 & E10 Hs.417549 NM_ 002827 PTPN1 Protein tyrosine phosphatase, non-receptor type 1 \\
\hline Ell & 24.45047379 & 25.67455482 & E11 Hs.446336 NM_002859 PXN Paxillin \\
\hline E12 & 21.3409214 & 23.70915985 & E12 Hs.413812 NM_006903 RAC1 Ras-related C3 botulinum toxin substrate 1 (rho family, small GIP binding protein Racl) \\
\hline F1 & 25.98708725 & 27.12110901 & F01 Hs.517601 NM 002872 RAC2 Ras-related C3 botulinum toxin substrate 2 (rho family, small GTP binding protein Rac2) \\
\hline F2 & $24.191600 \mathrm{~s}$ & 26.81167603 & F02 Hs.664080 NM_ 002890 RASA1 RAS p21 protein activator (GTPase activating protein) 1 \\
\hline F3 & 24.5975666 & 26.46269226 & F03 Hs.263671 NM_ 002906 RDX Radixin \\
\hline F4 & 29.60364151 & 34.12672043 & F04 Hs.247565 NM 000539 RHO Rhodopsin \\
\hline F5 & 21.28255463 & 23.98625374 & F05 Hs.247077 NM 001664 RHOA Ras homolog gene family, member A \\
\hline F6 & 30.70744514 & 31.91804886 & F06 Hs.502876 NM_004040 RHOB Ras homolog gene family, member B \\
\hline F7 & 23.5939064 & 25.41741562 & F07 Hs.502659 NM 175744 RHOC Ras homolog gene family, member C \\
\hline F8 & 23.17214012 & 24.90794182 & F08 Hs.6838 NMI 005168 RND3 Rho family GTPase 3 \\
\hline F9 & 24.386549 & 25.91716003 & F09 Hs.306307 NM 005406 ROCK1 Rho-associated, coiled-coil containing protein kinase 1 \\
\hline F10 & 28.26019287 & 32.3459816 & F10 Hs.594708 NM_014631 SH3PXD2A SH3 and PX domains 2A \\
\hline F11 & 26.79497337 & 27.70876503 & F1l Hs.195659 NM_ 005417 SRC V-src sarcoma (Schmidt-Ruppin A-2) viral oncogene homolog (avian) \\
\hline F12 & 37.13027954 & Undetermined & F12 Hs.463059 NM 003150 STAT3 Signal transducer and activator of transcription 3 (acute-phase response factor) \\
\hline G1 & 28.18871689 & 30.0135994 & G01 Hs.499209 NM 003174 SVIL Supervillin \\
\hline G2 & 27.76268196 & 28.59247398 & G02 Hs.645227 NM 000660 TGFB1 Transforming growth factor, beta 1 \\
\hline G3 & 25.40254402 & 26.19152451 & G03 Hs.633514 NM_ 003255 TMM2 TMMP metallopeptidase inhibitor 2 \\
\hline G4 & 28.89860725 & 31.82224846 & G04 Hs.471014 NM_006289 TLN1 Talin I \\
\hline G5 & 29.91687012 & 31.4691658 & G05 Hs.515469 NM 003370 VASP Vasodilator-stimulated phosphoprotein \\
\hline G6 & 23.40555 & 24.22704124 & G06 Hs.643896 NM 003373 VCL Vinculin \\
\hline G7 & 23.75560951 & 25.89518166 & G07 Hs.73793 NM_ 003376 VEGFA Vascular endothelial growth factor A \\
\hline GS & 18.81512833 & 21.32417107 & G08 Hs.642813 NM_ 003380 VMM Vimentin \\
\hline G9 & 24.60319138 & 27.27263641 & G09 Hs.75850 NM_ 003931 WASF1 WAS protein family, member 1 \\
\hline G10 & 24.80859566 & 26.20161247 & G10 Hs.590909 NM 006990 WASF2 WAS protein family, member 2 \\
\hline Gll & 28.88785172 & 32.18074417 & G11 Hs.143728 NM_ 003941 WASL Wiskott-Aldrich syndrome-like \\
\hline G12 & 30.14517593 & 34.10474396 & G12 Hs.128067 NM 003387 WIPF1 WAS/WASL interacting protein family, member 1 \\
\hline Hl & 20.80312157 & 22.01507187 & H01 Hs.520640 NM_ 001101 ACTB Actin, beta \\
\hline H2 & 19.84791946 & 21.98305702 & H02 Hs.534255 NM_ 004048 B2M Bet \\
\hline H3 & 20.16509628 & 22.14790154 & H03 Hs.592355 NM_ 002046 GAPDH Glyceraldehyde-3-phosphate dehydrogenase \\
\hline $\mathrm{H} 4$ & 22.88106728 & 24.81427956 & H04 HS.412707 NM 000194 HPRTI Hypoxanthine phosphoribosyltransferase 1 \\
\hline H5 & 16.50558662 & 18.6265316 & H05 Hs.546285 NM_ 001002 RPLP0 Ribosomal protein, large, P0 \\
\hline H6 & 29.709795 & 35.55280304 & H06 N/A SA_00105 HGDC Human Genomic DNA Contamination \\
\hline H7 & 21.19672394 & 22.13027573 & H07 N/A SA_00104 RTC Reverse Transcription Control \\
\hline H8 & 21.18412781 & 22.08263016 & H0S N/A SA 00104 RTC Reverse Transcription Control \\
\hline Ho & 21.25595093 & 22.09686661 & H09 N/A SA_00104 RTC Reverse Transcription Control \\
\hline milo & 18.93539429 & 22.68627167 & H10 N/A SA_00103 PPC Positive PCR Control \\
\hline Hil & 19.14422035 & 22.74818039 & HIl N/A SA_00103 PPC Positive PCR Control \\
\hline & 18.97528267 & & \\
\hline
\end{tabular}



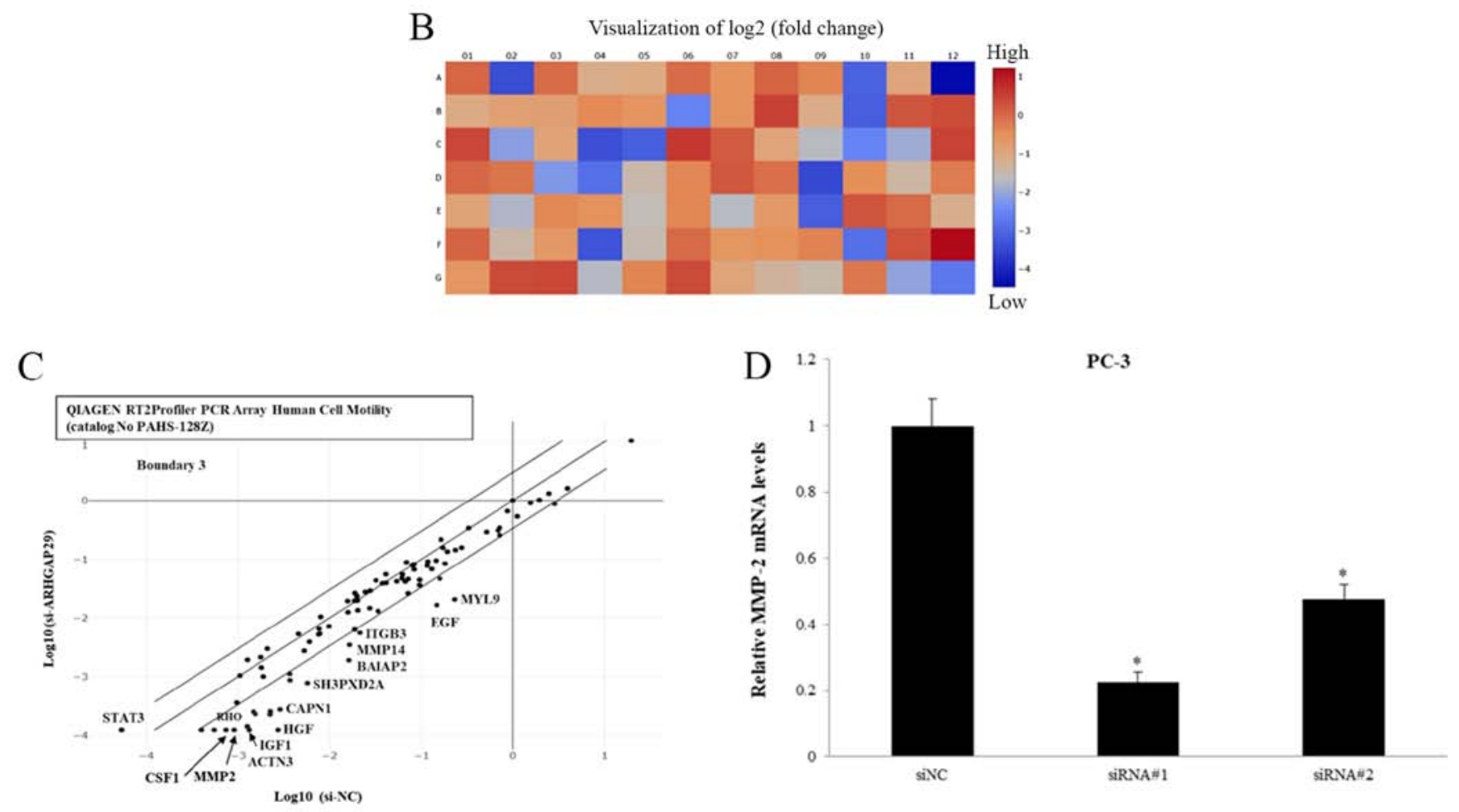

Figure 4. Identification of other cell motility-related genes after knockdown of ARHGAP29. (A) The 84 genes related to cell motility are presented and analysis of the results was performed using the web-based software 'RT2 Profiler PCR Array' Data Analysis version 3.5. All of genes involved in the array are presented. (B) A heatmap is presented. Numerous genes were downregulated after knockdown of ARHGAP29 in PC3 cells. A1-G12 corresponds to A1-G12 of A. In the heatmap the data was displayed in a grid where each row represents a gene included in the commercial array. The color and intensity of the boxes represent changes of gene expression. For example, red represents upregulated genes and blue represents downregulated genes. (C) PCR Array of cell motility genes in PC-3 cells before and after downregulation of ARHGAP29. The graph shows the relative log value between cell motility gene expression in PC-3 cells before and after downregulation of ARHGAP29 (vertical axis, after downregulation of ARHGAP29; horizontal axis, before downregulation of ARHGAP29). The diagonal line in the center of the graph shows equal level of gene expression in PC-3 cells before and after downregulation of ARHGAP29. The upper diagonal lines indicate that the level of gene expression after downregulation was three times higher than that of before downregulation. The lower diagonal line indicates that the level of gene expression after downregulation was one third times lower than that of before downregulation. The mRNA level of MMP-2 was significantly downregulated. (D) Quantitative comparison of MMP-2 by RT-qPCR between si-NC and si-ARHGAP29 PC-3 transfectants. MMP-2 mRNA expression was suppressed after downregulation of ARHGAP29. Experiments were performed in triplicate. The results represent the mean \pm SD. "P<0.01 compared with si-NC. ARHGAP29, Rho GTPase-activating protein 29.

Table II. Univariate and multivariate analyses of prognostic factors associated with biochemical recurrence-free survival of prostate cancer patients.

\begin{tabular}{|c|c|c|c|c|}
\hline & \multicolumn{2}{|c|}{ Univariate analysis } & \multicolumn{2}{|c|}{ Multivariate analysis } \\
\hline & $\operatorname{HR}(95 \% \mathrm{CI})$ & P-value & HR $(95 \% \mathrm{CI})$ & P-value \\
\hline \multicolumn{5}{|l|}{ Initial PSA* (ng/ml) } \\
\hline$<20$ vs. $\geq 20$ & $0.53(0.30-1.00)$ & 0.049 & $0.37(0.15-0.88)$ & 0.025 \\
\hline \multicolumn{5}{|l|}{ D'Amico risk classification } \\
\hline Low, intermediate vs. high & $0.73(0.43-1.24)$ & 0.248 & & \\
\hline \multicolumn{5}{|l|}{ Gleason score } \\
\hline$<8$ vs. $\geq 8$ & $0.84(0.47-1.57)$ & 0.567 & & \\
\hline \multicolumn{5}{|l|}{ Pathological T category } \\
\hline$<\mathrm{pT} 2 \mathrm{c}$ vs. $\geq \mathrm{pT} 2 \mathrm{c}$ & $0.60(0.30-1.10)$ & 0.099 & & \\
\hline \multicolumn{5}{|l|}{ Expression of YAP } \\
\hline Low vs. high & $0.53(0.28-1.01)$ & 0.052 & & \\
\hline \multicolumn{5}{|l|}{ Expression of ARHGAP29 } \\
\hline Low vs. high & $0.46(0.24-086)$ & 0.015 & $0.44(0.19-0.95)$ & 0.037 \\
\hline
\end{tabular}

HR, hazard ratio; CI, confidence interval; PSA, prostate specific antigen; YAP, yes-associated protein; ARHGAP29, Rho GTPase-activating protein 29 . 


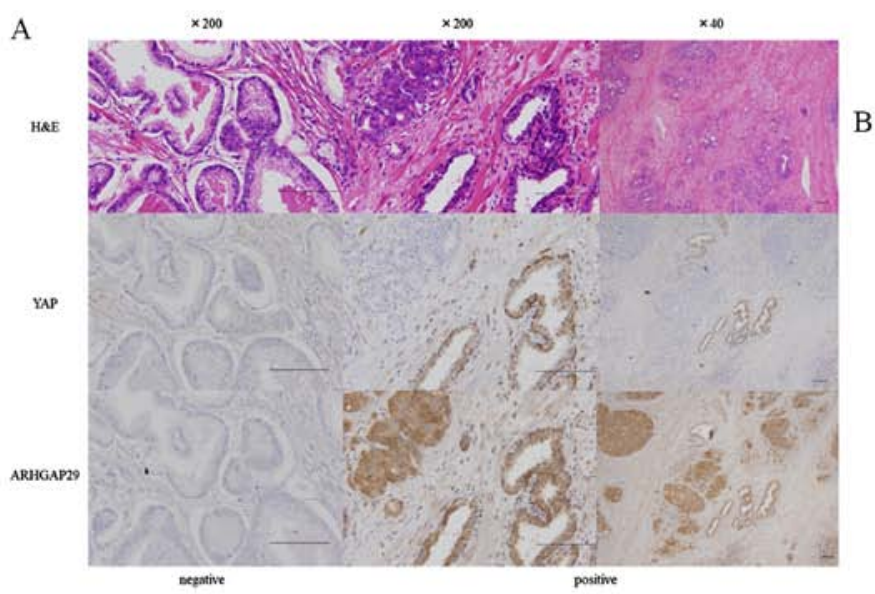

B
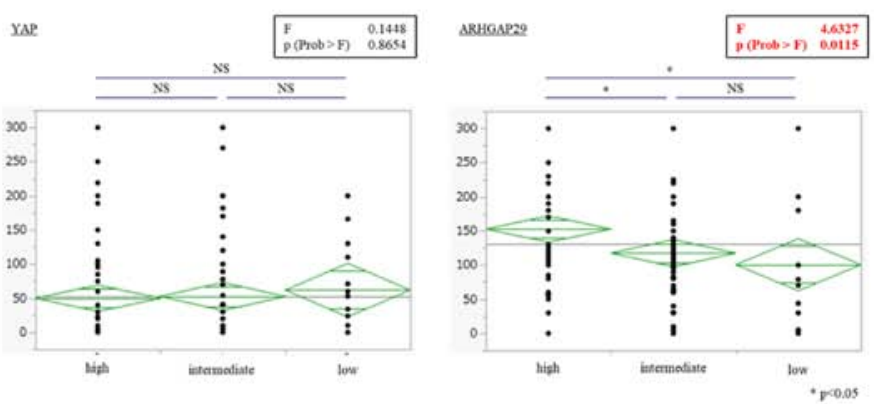

C
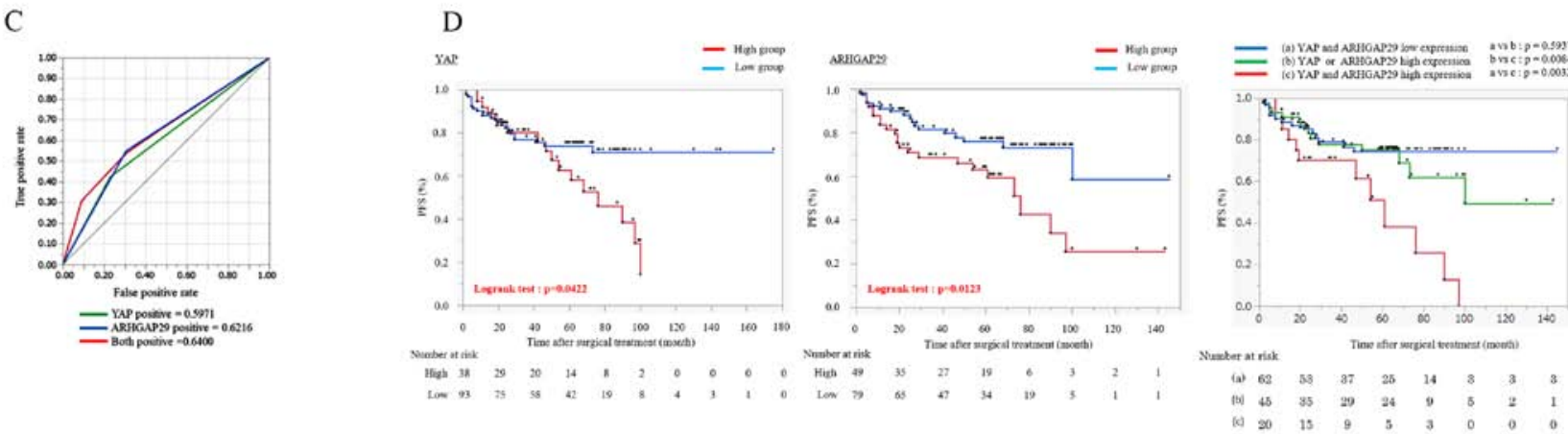

Figure 5. Association between the expression levels of YAP and ARHGAP29 and the prognosis of prostate cancer patients. (A) Histology (H\&E staining) and IHC staining of YAP or ARHGAP29. Protein expression patterns were different between YAP and ARHGAP29. YAP was heterogeneously stained. Scale bars represent $200 \mu \mathrm{M}$. (B) Association of the D'Amico risk classification with the expression of YAP and ARHGAP29. When comparing three groups, one-way ANOVA (inside the black frame) followed by Tukey-Kramer test were used. "P<0.05. YAP was unrelated to the risk classification, but ARHGAP29 was significantly associated with the risk classification. The diamond indicates the mean (long horizontal line) and $95 \%$ confidence interval of the $\mathrm{H}$-score. (C) ROC curve of YAP, ARHGAP29 and both (AUC: 0.5971, 0.6216 and 0.6400, respectively). Both proteins had low AUC scores as prognostic markers. (D) Kaplan-Meier plot of biochemical PFS stratified by the expression levels of YAP, ARHGAP29 and both. For each protein, high expression was associated with a poor prognosis of prostate cancer patients. Furthermore, the group with high expression of both YAP and ARHGAP29 had the worst prognosis. PFS was compared by a log-rank test. $\mathrm{P}<0.05$ was considered to indicate a statistically significant difference. YAP, yes-associated protein; ARHGAP29, Rho GTPase-activating protein 29; ROC, receiver operating characteristic; AUC, area under the curve; PFS, progression-free survival; NS, not significant.

lung cancers as well as melanoma (25-31). Similar to other cancers, YAP regulates cell migration and invasion in prostate cancer (13). Several ARHGAPs, which enhance Rho GTPase activity in almost all basic cellular processes, are oncogenic or tumor suppressor proteins (32). For example, ARHGAP5 and ARHGAP42 have been revealed to be oncogenic proteins in nasopharyngeal cancer $(33,34)$, whereas ARHGAP24 has been demonstrated to be a tumor suppressor protein in lung, breast, and colorectal cancers (35-38). Numerous studies have demonstrated a close association of ARHGAPs with several malignancies.

Recently, other ARHGAPs such as ARHGAP18 and ARHGAP29 $(17,18)$ were identified as transcriptional targets of YAP, and ARHGAP29 was reported as a prognostic marker for gastric cancer. Since there have been no studies on ARHGAP29 in prostate cancer, in the present study, ARHGAP29 was examined to investigate how it affects progression or metastasis of prostate cancer and whether ARHGAP29 may be a prognostic marker for prostate cancer. Initially, protein expression in four prostate cancer cell lines (22Rv1, LNCaP, DU145 and PC-3) was assessed. Among these cell lines, PC-3 and DU145 did not express AR, but highly expressed YAP. In contrast, YAP expression was low in AR-expressing cell lines
(22Rv1 and LNCaP). PC-3 cells highly expressed ARHGAP29 compared with the other three cell lines. AR-null PC-3 cells are derived from bone metastasis $(39,40)$. After complete knockdown of ARHGAP29 in PC-3 cells, their proliferation and invasion were significantly decreased. In contrast, cell proliferation and invasion were increased after upregulation of ARHGAP29 in LNCaP and DU145 cells. In the present study, we did not investigate a direct interaction between AR and ARHGAP29. However, the present results indicated that ARHGAP29 regulates cell proliferation and invasion in prostate cancer cells. Recently, Qiao et al demonstrated that ARHGAP29 suppressed the RhoA-cofilin pathway and destabilized F-actin, which caused cytoskeletal rearrangement and promoted migration (18). In the present study, certain proteins in the RhoA-cofilin pathway were analyzed in PC-3, LNCaP and DU145 cells. Specifically, phosphorylated cofilin and F-actin were recovered when ARHGAP29 was completely knocked down in PC-3 cells. Moreover, the relative protein level of phosphorylated cofilin to cofilin was increased. These data were consistent with the results from a recent study on a gastric cancer cell line (18). F-actin was slightly decreased in DU145 cells following upregulation of ARHGAP29. In PC-3 cells, ARHGAP29 may be associated with cell migration by 
suppressing the RhoA-cofilin pathway similar to a previous study (18). However, cofilin and p-cofilin expression were not altered in LNCaP and DU145 cells. Specifically, the results of LNCaP and Du145 cells were not demonstrated as the reverse of the observations made in PC-3 ARHGAP29-knockdown cells. These results may be explained by the fact that each cell line has a different genotype/phenotype as revealed in a previous study (for instance only PC-3 cells do not express $\alpha$-catenin) (40). Upregulation of ARHGAP29 may lead to decrease of F-actin via another pathway in LNCaP and DU145 cells, however, to demonstrate this, further experiments are required.

Apart from the Rho-A-cofilin pathway, to identify new targets or cancer pathways related to ARHGAP29, a pre-designed array (Human Cell Motility), based on the functional analysis data in the present study, was used. Among the 84 genes in the array, expression of several genes was altered after knocking down ARHGAP29 in PC-3 cells. Among the genes, expression of MMP-2 was validated by RT-qPCR and western blotting. Among the matrix metalloproteinase (MMP) family, which degrade the ECM, MMP-2, also known as gelatinase A, is reported to be correlated with the invasion and metastasis of cancer cells as well as angiogenesis in numerous human cancer tissues $(41,42)$. Moreover, Zhang et al indicated a role of YAP in gastric cancer and revealed that LATS1 inhibited the growth and metastasis of gastric cancer cells by restraining nuclear transfer of YAP and downregulating MMP-2 expression concurrently (43). This suggests that the YAP pathway, which regulates the progression of cancer cells, is associated with MMP-2. In previous studies on prostate cancer development, it has been similarly demonstrated that MMP-2 is associated with invasion, metastasis, and a poor prognosis (44-46). It is theorized that ARHGAP29 may activate cell motility to upregulate MMP-2. In the present, we did not establish a direct interaction between ARGAP29 and MMP-2. Therefore, further experiments are required to support this theory.

Next, IHC staining was performed to investigate the clinical role of YAP and ARHGAP29 protein expression in prostate cancer patients. High expression levels of ARHGAP29 were related to the D'Amico risk classification, which is the risk classification of prostate cancer, and prognosis of prostate cancer patients (PSA PFS). In the present study, prostate cancer patients with high YAP or ARHGAP29 expression had a significantly poor prognosis. These differences between the TCGA database and our data may be due to different characteristics of the cohort including racial bias. Based on our data, ARHGAP29 may be a prognostic marker and therapeutic target. To confirm the present results, large-scale data analysis using Japanese samples is required in the future.

A limitation of the present study is the lack of co-localization studies of AR, YAP and ARHGAP29 in human prostate specimens as well as lack of ARHGAP29 rescue experiments in prostate cancer cells.

In conclusion, it was demonstrated that ARHGAP29 may be associated with prostate cancer cell growth and invasion as well as a clinically poor prognosis of prostate cancer patients. Therefore, ARHGAP29 may serve as a new biomarker or novel therapeutic target in prostate cancer. In a future study, the investigation of the relationship between YAP and the ARHGAP29 pathway is required to elucidate the underlying mechanism in prostate cancer.

\section{Acknowledgements}

We thank Kiyomi Fujita and Takao Kitagawa for technical assistance.

\section{Funding}

The present study was funded by a Grant-in-Aid for Scientific Research (C) (KAKENHI-PROJECT-16K11008).

\section{Availability of data and materials}

The datasets used and/or analyzed during the current study are available from the corresponding author on reasonable request.

\section{Authors' contributions}

HMatsum, HH, MFS and HMatsuy conceived and designed the study. All authors advised the work. KS, HH and KU performed experiments. KS, MS and $\mathrm{HH}$ prepared the figures and drafted the original manuscript. JM, NF, YK, RI, YY, SY and TS contributed to the analysis or interpretation of the data. HMatsum, HH and HMatsuy reviewed and revised the manuscript. All authors read and approved the final manuscript.

\section{Ethics approval and consent to participate}

The present study was approved by the Institutional Ethics Committee of the Graduate School of Medicine of Yamaguchi University and written informed consent was obtained from all individuals enrolled in the study.

\section{Patient consent for publication}

Not applicable.

\section{Competing interests}

The authors declare that they have no competing interests.

\section{References}

1. Bray F, Ferlay J, Soerjomataram I, Siegel RL, Torre LA and Jemal A: Global cancer statistics 2018: GLOBOCAN estimates of incidence and mortality worldwide for 36 cancers in 185 countries. CA Cancer J Clin 68: 394-424, 2018.

2. Pagliuca M, Buonerba C, Fizazi K and Di Lorenzo G: The evolving systemic treatment landscape for patients with advanced prostate cancer. Drugs 79: 381-400, 2019.

3. Xu J, Zheng SL, Komiya A, Mychaleckyj JC, Isaacs SD, Hu JJ, Sterling D, Lange EM, Hawkins GA, Turner A, et al: Germline mutations and sequence variants of the macrophage scavenger receptor 1 gene are associated with prostate cancer risk. Nat Genet 32: 321-325, 2002.

4. Jefferies MT, Cox AC, Shorning BY, Meniel V, Griffiths D, Kynaston HG, Smalley MJ and Clarke AR: PTEN loss and activation of K-RAS and $\beta$-catenin cooperate to accelerate prostate tumourigenesis. J Pathol 243: 442-456, 2017.

5. Suzuki H, Freije D, Nusskern DR, Okami K, Cairns P, Sidransky D, Isaacs WB and Bova GS: Interfocal heterogeneity of PTEN/MMAC1 gene alterations in multiple metastatic prostate cancer tissues. Cancer Res 58: 204-209, 1998.

6. Suzuki H, Komiya A, Aida S, Ito H, Yatani R and Shimazaki J: Detection of human papillomavirus DNA and p53 gene mutations in human prostate cancer. Prostate 28: 318-324, 1996. 
7. Grignon DJ, Caplan R, Sarkar FH, Lawton CA, Hammond EH, Pilepich MV, Forman JD, Mesic J, Fu KK, Abrams RA, et al: p53 status and prognosis of locally advanced prostatic adenocarcinoma: A study based on RTOG 8610. J Natl Cancer Inst 89: 158-165, 1997.

8. Zhou Z, Flesken-Nikitin A, Corney DC, Wang W, Goodrich DW, Roy-Burman P and Nikitin AY: Synergy of p53 and Rb deficiency in a conditional mouse model for metastatic prostate cancer. Cancer Res 66: 7889-7898, 2006.

9. Dong JT, Li CL, Sipe TW and Frierson HF Jr: Mutations of PTEN/MMAC1 in primary prostate cancers from Chinese patients. Clin Cancer Res 7: 304-308, 2001.

10. Wang SI, Parsons R and Ittmann M: Homozygous deletion of the PTEN tumor suppressor gene in a subset of prostate adenocarcinomas. Clin Cancer Res 4: 811-815, 1998.

11. Lin HK, Hu YC, Lee DK and Chang C: Regulation of androgen receptor signaling by PTEN (phosphatase and tensin homolog deleted on chromosome 10) tumor suppressor through distinct mechanisms in prostate cancer cells. Mol Endocrinol 18: 2409-2423, 2004.

12. Nelson PS: Molecular states underlying androgen receptor activation: A framework for therapeutics targeting androgen signaling in prostate cancer. J Clin Oncol 30: 644-646, 2012.

13. Zhang L, Yang S, Chen X, Stauffer S, Yu F, Lele SM, Fu K, Datta K, Palermo N, Chen Y, et al: The hippo pathway effector YAP regulates motility, invasion, and castration-resistant growth of prostate cancer cells. Mol Cell Biol 35: 1350-1362, 2015.

14. Noh MG,Kim SS,Hwang EC,Kwon DD and Choi C: Yes-associated protein expression is correlated to the differentiation of prostate adenocarcinoma. J Pathol Transl Med 51: 365-373, 2017.

15. Collak FK, Demir U, Ozkanli S, Kurum E and Zerk PE: Increased expression of YAP1 in prostate cancer correlates with extraprostatic extension. Cancer Biol Med 14: 405-413, 2017.

16. Jin $\mathrm{X}$, Zhao W, Zhou P and Niu T: YAP knockdown inhibits proliferation and induces apoptosis of human prostate cancer DU145 cells. Mol Med Rep 17: 3783-3788, 2018.

17. Porazinski S, Wang H, Asaoka Y, Behrndt M, Miyamoto T, Morita H, Hata S, Sasaki T, Krens SFG, Osada Y, et al: YAP is essential for tissue tension to ensure vertebrate 3D body shape. Nature 521: 217-221, 2015.

18. Qiao Y, Chen J,Lim YB, Finch-Edmondson ML, Seshachalam VP, Qin L, Jiang T, Low BC, Singh H, Lim CT, et al: YAP regulates actin dynamics through ARHGAP29 and promotes metastasis Cell Rep 19: 1495-1502, 2017.

19. Dupont S, MorsutL, Aragona M,Enzo E, Giulitti S, Cordenonsi M, Zanconato, Le Digabel J, Forcato M, Bicciato S, et al: Role of YAP/TAZ in mechanotransduction. Nature 474: 179-183, 2011.

20. Goulding H, Pinder S, Cannon P, Pearson D, Nicholson R, Snead D, Bell J, Elston CW, Robertson JF, Blamey RW, et al: A new immunohistochemical antibody for the assessment of estrogen receptor status on routine formalin-fixed tissue samples. Hum Pathol 26: 291-294, 1995.

21. Ishibashi H, Suzuki T, Suzuki S, Moriya T, Kaneko C, Takizawa T, Sunamori M, Handa M, Kondo T and Sasano H: Sex steroid hormone receptors in human thymoma. J Clin Endocrinol Metab 88: 2309-2317, 2003.

22. Pirker R, Pereira JR, von Pawel J, Krzakowski M, Ramlau R, Park K, de Marinis F, Eberhardt WE, Paz-Ares L, Störkel S, et al: EGFR expression as a predictor of survival for first-line chemotherapy plus cetuximab in patients with advanced non-small-cell lung cancer: Analysis of data from the phase 3 FLEX study. Lancet Oncol 13: 33-42,2012.

23. Livak KJ and Schmittgen TD: Analysis of relative gene expression data using real-time quantitative PCR and the 2(-Delta Delta C(T)) method. Methods 25: 402-408, 2001.

24. Kawakami T, Takeuchi S, Arimura Y and Soma Y: Elevated antilysosomal-associated membrane protein-2 antibody levels in patients with adult Henoch-Schönlein purpura. Br J Dermatol 166: 1206-1212, 2012

25. Pan Z, Tian Y, Zhang B, Zhang X, Shi H, Liang Z, Wu P, Li R, You B, Yang L, et al: YAP signaling in gastric cancer-derived mesenchymal stem cells is critical for its promoting role in cancer progression. Int J Oncol 51: 1055-1066, 2017.

26. Chen D, Sun Y, Wei Y, Zhang P, Rezaeian AH Teruya-Feldstein J, Gupta S, Liang H, Lin HK, Hung MC, et al: LIFR is a breast cancer metastasis suppressor upstream of the Hippo-YAP pathway and a prognostic marker. Nat Med 18 $1511-1517,2012$
27. Lamar JM, Stern P, Liu H, Schindler JW, Jiang ZG and Hynes RO: The Hippo pathway target, YAP, promotes metastasis through its TEAD-interaction domain. Proc Natl Acad Sci USA 109: E2441-E2450, 2012

28. Xu MZ, Chan SW, Liu AM, Wong KF, Fan ST, Chen J, Poon RT, Zender L, Lowe SW, Hong W, et al: AXL receptor kinase is a mediator of YAP-dependent oncogenic functions in hepatocellular carcinoma. Oncogene 30: 1229-1240, 2011.

29. Zhang M, Zhao Y, Zhang Y, Wang D, Gu S, Feng W, Peng W, Gong $\mathrm{A}$ and $\mathrm{Xu} \mathrm{M}$ : LncRNA UCA1 promotes migration and invasion in pancreatic cancer cells via the Hippo pathway. Biochim Biophys Acta Mol Basis Dis 1864: 1770-1782, 2018.

30. Hsu PC, Miao J, Huang Z, Yang YL, Xu Z, You J, Dai Y, Yeh CC, Chan G, Liu S, et al: Inhibition of yes-associated protein suppresses brain metastasis of human lung adenocarcinoma in a murine model. J Cell Mol Med 22: 3073-3085, 2018.

31. Jin D, Guo J, Wang D, Wu Y, Wang X, Gao Y, Shao C, Xu X and Tan S: The antineoplastic drug metformin downregulates YAP by interfering with IRF-1 binding to the YAP promoter in NSCLC. EBioMedicine 37: 188-204, 2018.

32. Tcherkezian $\mathrm{J}$ and Lamarche-Vane $\mathrm{N}$ : Current knowledge of the large RhoGAP family of proteins. Biol Cell 99: 67-86, 2007.

33. Fang Y, Zhu X, Wang J, Li N, Li D, Sakib N, Sha Z and Song W: MiR-744 functions as a proto-oncogene in nasopharyngeal carcinoma progression and metastasis via transcriptional control of ARHGAP5. Oncotarget 6: 13164-13175, 2015.

34. Hu Q, Lin X, Ding L, Zeng Y, Pang D, Ouyang N, Xiang Y and Yao H: ARHGAP42 promotes cell migration and invasion involving PI3K/Akt signaling pathway in nasopharyngeal carcinoma. Cancer Med 7: 3862-3874, 2018.

35. Wang L, Shen S, Wang M, Ding F, Xiao H, Li G and Hu F: Rho GTPase activating protein 24 (ARHGAP24) silencing promotes lung cancer cell migration and invasion by activating $\beta$-catenin signaling. Med Sci Monit 25: 21-31, 2019

36. Dai X, Geng F, Dai J, Li M and Liu M: Rho GTPase activating Protein 24 (ARHGAP24) regulates the anti-cancer activity of sorafenib against breast cancer MDA-MB-231 cells via the signal transducer and activator of transcription 3 (STAT3) signaling pathway. Med Sci Monit 24: 8669-8677, 2018.

37. Uehara S, Saito K, Asami H and Ohta Y: Role of ARHGAP24 in ADP ribosylation factor 6 (ARF6)-dependent pseudopod formation in human breast carcinoma cells. Anticancer Res 37: 4837-4844, 2017.

38. Zhang S, Sui L, Zhuang J, He S, Song Y, Ye Y and Xia W: ARHGAP24 regulates cell ability and apoptosis of colorectal cancer cells via the regulation of P53. Oncol Lett 16: 3517-3524, 2018.

39. Kaighn ME, Narayan KS, Ohnuki Y, Lechner JF and Jones LW: Establishment and characterization of a human prostatic carcinoma cell line (PC-3). Invest Urol 17: 16-23, 1979.

40. Mitchell S, Abel P, Ware M, Stamp G and Lalani E: Phenotypic and genotypic characterization of commonly used human prostatic cell lines. BJU Int 85: 932-944, 2000.

41. Li Y, Song T, Chen Z, Wang Y, Zhang J and Wang X: Pancreatic stellate cells activation and matrix metallopeptidase 2 expression correlate with lymph node metastasis in pancreatic carcinoma. Am J Med Sci 357: 16-22, 2019.

42. Maekawa R, Maki H, Yoshida H, Hojo K, Tanaka H, Wada T, Uchida N, Takeda Y, Kasai H, Okamoto H, et al: Correlation of antiangiogenic and antitumor efficacy of N-biphenyl sulfonyl-phenylalanine hydroxiamic acid (BPHA), an orally-active, selective matrix metalloproteinase inhibitor. Cancer Res 59: 1231-1235, 1999.

43. Zhang J, Wang G, Chu SJ, Zhu JS, Zhang R, Lu WW, Xia LQ, $\mathrm{Lu} \mathrm{YM}, \mathrm{Da}$ W and Sun Q: Loss of large tumor suppressor 1 promotes growth and metastasis of gastric cancer cells through upregulation of the YAP signaling. Oncotarget 7: 16180-16193, 2016.

44. Trudel D, Fradet Y, Meyer F, Harel F and Têtu B: Significance of MMP-2 expression in prostate cancer: An immunohistochemical study. Cancer Res 63: 8511-8515, 2003.

45. Chen PC, Tang CH, Lin LW, Tsai CH, Chu CY, Lin TH and Huang YL: Thrombospondin-2 promotes prostate cancer bone metastasis by the up-regulation of matrix metalloproteinase- 2 through down-regulating miR-376c expression. J Hematol Oncol 10: 33, 2017

46. Ito Y, Ishiguro H, Kobayashi N, Hasumi H, Watanabe M, Yao M and Uemura H: Adipocyte-derived monocyte chemotactic protein-1 (MCP-1) promotes prostate cancer progression through the induction of MMP-2 activity. Prostate 75: 1009-1019, 2015. 